\title{
O uso da cirurgia bariátrica no controle do diabetes tipo 2 em pacientes com índice de massa corpórea $\leq 35 \mathrm{~kg} / \mathrm{m}^{2}$
}

The use of bariatric surgery to control type 2 diabetes in body mass index patients $\leq 35 \mathrm{~kg} / \mathrm{m}^{2}$

El uso de cirugía bariátrica para controlar la diabetes tipo 2 en pacientes con índice de masa corporal $\leq 35 \mathrm{~kg} / \mathrm{m}^{2}$

Bráulio Filgueira Magalhães ${ }^{1 *}$, Pedro Hugo Bezerra Maia Filho ${ }^{1}$, George Wallisson Severo De Sá ${ }^{2}$, Whallyson Pinheiro Mascarenhas ${ }^{2}$, Igor de Morais Marques² ${ }^{2}$, Lucas Benevides Maia², Stenio Santos Moura $^{2}$, Ticiane Ponciano de Oliveira Lima², Gabriella Rocha Moreira ${ }^{1}$, Yanny Brena Alencar Araújo ${ }^{1}$, Hermes Melo Teixeira Batista ${ }^{1}$, Thyciara Fontenele Marques ${ }^{1}$.

\section{RESUMO}

Objetivo: Explicar a possibilidade da cirurgia bariátrica como alternativa terapêutica no controle do Diabetes Mellitus tipo 2 em pacientes com índice de massa corpórea abaixo de $35 \mathrm{~kg} / \mathrm{m}^{2}$. Métodos: Trata-se de uma Revisão Sistemática com base em estudos randomizados e não randomizados, sobre a efetividade da cirurgia bariátrica no controle do diabetes tipo 2 em obesos leves. Foi realizada uma busca de artigos publicados em periódicos internacionais nas bases de dados: SCOPUS, Web Of Science e PubMed indexados de 2013 a 2017. Resultados: O DM 2 é um grave problema mundial de saúde pública e suas complicações microvasculares e cardiovasculares aumentam substancialmente a morbidade e a mortalidade. Hoje, a cirurgia bariátrica é a terapia de emagrecimento mais eficaz disponível e possibilita atingir níveis glicêmicos adequados em pacientes com DM2 ainda com reserva de células beta pancreáticas. Considerações finais: Concluiu-se que as pesquisas sobre o tema são recentes, sendo a mais longa efetuada num período de sete anos, seus resultados no geral mostram excelentes taxas de controle no primeiro ano de seguimento, porém conforme o passar do tempo há ainda menos estudos que acompanharam esses pacientes, e os seus resultados decaem, não sendo possível efetivamente corroborar com seus achados iniciais.

Palavras-chave: Diabetes tipo 2, Obesidade, Cirurgia Bariátrica.

\begin{abstract}
Objective: Explain the possibility of bariatric surgery as a therapeutic alternative in the control of type 2 diabetes mellitus in patients with body mass index below $35 \mathrm{~kg} / \mathrm{m} 2$. Methods: This is a Systematic Review based on randomized and nonrandomized studies on the effectiveness of bariatric surgery in controlling type 2 diabetes in mildly obese. We searched articles published in international journals in the databases: SCOPUS, Web Of Science and PubMed indexed from 2013 to 2017. Results: DM 2 is a serious worldwide public health problem and its microvascular and cardiovascular complications substantially increase morbidity and mortality. Today, bariatric surgery is the most effective weight loss therapy available and enables adequate glycemic levels to be achieved in patients with T2DM still with pancreatic beta cell reserve. Final considerations: It is concluded that research on the subject is recent, the longest being carried out over a period of seven years, its results generally show excellent control rates in the first year of follow-up, but as time goes by there are even fewer studies that have followed up. these patients, and their results decline, and cannot effectively corroborate their initial findings.
\end{abstract}

Key words: Type 2 diabetes, Obesity, Bariatric Surgery.

\footnotetext{
${ }^{1}$ Faculdade de Medicina Estácio de Juazeiro do Norte - ESTÁCIO FMJ, Juazeiro do Norte-CE.

*E-mail: braulio.magalhaes@hotmail.com

${ }^{2}$ Hospital Regional do Cariri (HRC), Juazeiro do Norte-CE.
}

SUBMETIDO EM: $9 / 2019$

ACEITO EM: 10/2019

PUBLICADO EM: 10/2019 


\section{RESUMEN}

Objetivo: Explicar la posibilidad de la cirugía bariátrica como alternativa terapéutica en el control de la diabetes mellitus tipo 2 en pacientes con un índice de masa corporal inferior a $35 \mathrm{~kg} / \mathrm{m} 2$. Métodos: Esta es una revisión sistemática basada en estudios aleatorios y no aleatorios sobre la efectividad de la cirugía bariátrica en el control de la diabetes tipo 2 en personas con obesidad leve. Se realizaron búsquedas en artículos publicados en revistas internacionales en las bases de datos: SCOPUS, Web Of Science y PubMed indexados de 2013 a 2017. Resultados: DM 2 es un grave problema de salud pública en todo el mundo y sus complicaciones microvasculares y cardiovasculares aumentan sustancialmente la morbilidad y la mortalidad. Hoy en día, la cirugía bariátrica es la terapia de pérdida de peso más efectiva disponible y permite alcanzar niveles glucémicos adecuados en pacientes con DM2 que todavía tienen reserva de células beta pancreáticas. Consideraciones finales: Se concluye que la investigación sobre el tema es reciente, la más larga llevada a cabo en un período de siete años, sus resultados generalmente muestran excelentes tasas de control en el primer año de seguimiento, pero a medida que pasa el tiempo hay aún menos estudios que han seguido estos pacientes, y sus resultados disminuyen, y no pueden corroborar efectivamente sus hallazgos iniciales.

Palabras clave: Diabetes tipo 2, obesidad, cirugía bariátrica

\section{INTRODUÇÃO}

O Diabetes Mellitus tipo 2 (DM2) é considerado um grave problema de saúde púbica mundial pela sua alta prevalência e efeitos na qualidade de vida (CHONDRONIKOLA M, et al., 2016). Atualmente acomete aproximadamente 600 milhões de pessoas no mundo e apresenta uma taxa de 8,9\% no Brasil (BRASIL, 2016; ELIGAR VS e NARAYANASWAMY AKP, 2016). É caracterizada por uma progressiva disfunção nas células beta pancreáticas, aumento da resistência insulínica ou ambos (RUBINO F e AMIEL SA, 2014; KHANNA V e KASHYAP SR, 2015). O DM2 quando não tratada ou descontrolada, resulta em morbidade e mortalidade devido ao aumento do risco cardiovascular e complicações específicas como falência renal, retinopatia e neuropatia (CHONDRONIKOLA M, et al., 2016).

O tratamento do DM2 apresenta como objetivo reduzir a progressão da doença, pelo controle da hiperglicemia, hipertensão, dislipidemia e outros fatores de risco cardiovasculares (KHANNA V e KASHYAP SR, 2015), envolvendo terapia medicamentosa e mudanças no estilo de vida. Apesar das inúmeras drogas antidiabéticas, os pacientes continuam falhando no controle dos níveis glicêmicos, sendo a perda de peso o que tem efeito benéfico profundo nas anormalidades metabólicas envolvidas na fisiopatologia da doença (CHONDRONIKOLA M, et al., 2016). Muitas medicações são associadas à hipoglicemia e ganho ponderal especialmente na intensificação do tratamento, os episódios de hipoglicemia estão associados com maior incidência de mortes de causa cardiovascular em tratamento intensivo e o ganho de peso pode guiar uma futura deterioração no controle glicêmico (GANGULY S, et al, 2015).

$\mathrm{O}$ aumento da prevalência do DM2 é associado à pandemia de obesidade, o maior fator de risco para a doença (KHANNA V e KASHYAP SR, 2015). Mudanças no estilo de vida e o tratamento farmacológico da obesidade tem eficácia limitada, tanto na promoção de perda ponderal quanto na melhora das complicações metabólicas (MALKANI S, 2015).

A cirurgia bariátrica é atualmente a terapia de emagrecimento mais eficaz disponível, promovendo uma maior perda de peso sustentada, $20 \%$ a $30 \%$ após dois anos, e paralelamente, é considerada a melhor para atingir níveis glicêmicos adequados em pacientes com DM2 (CHONDRONIKOLA M, et al., 2016). No entanto, não há estudos de longo prazo e de alta qualidade sobre a melhoria das complicações micro e macrovasculares. Além disso, há existe uma remissão do diabetes ao longo do tempo, com 35 a $50 \%$ dos pacientes que inicialmente conseguiram remissão da doença apresentam recorrência do quadro com o passar do tempo (RUBINO F, et al., 2016).

Os procedimentos realizados para bariátrica são em sua maioria laparoscópicos e incluem: Bypass gástrico em Y de Roux, gastrectomia vertical com interposição ileal laparoscópica, banda gástrica ajustável laparoscópica, derivação bilio-pancreática. As cirurgias com bypass desviam os nutrientes distalmente no trato gastro-intestinal, enquanto as outras causam restrição ao fluxo normal da comida sem procedimentos 
restritivos (MALKANI S, 2015). Associação Americana de Diabetes (ADA - American Diabetes Association) e a Federação Internacional de Diabetes (IDF - International Diabetes Federation) recomendam a cirurgia bariátrica em seus guidelines para pacientes obesos de índice de massa corpórea (IMC) $>35 \mathrm{~kg} / \mathrm{m}^{2}$ (kilogram/ square meter) com DM2, afirmam que para pacientes com IMC $\leq 35 \mathrm{~kg} / \mathrm{m}^{2}$ a evidência de recomendação é insuficiente quando pacientes fora de protocolos de pesquisa (COELHO D, et al., 2018).

Dado o surgimento de alguns estudos que advogam a efetividade do uso de cirurgia bariátrica para pacientes obesos com diabetes como comorbidade, esta revisão sistemática busca estabelecer se esse tipo de cirurgia pode ser utilizada como opção terapêutica no controle do Diabetes Mellitus tipo 2 em pacientes com IMC $<35 \mathrm{~kg} / \mathrm{m}^{2}$.

\section{MÉTODOS}

Trata-se de uma Revisão Sistemática no qual foram utilizadas "a priori" para o protocolo do estudo, as seguintes etapas: a) definição da pergunta de revisão; b) critérios de inclusão e exclusão; c) estratégias para a busca dos estudos; d) seleção do material; e) análise crítica dos estudos e f) coleta e síntese dos dados.

A pesquisa foi norteada a partir da seguinte questão: A cirurgia bariátrica pode ser, a partir dos seus impactos metabólicos no organismo do individuo, uma opção terapêutica no controle do diabetes mellitus tipo 2 em pacientes que apresentam obesidade grau 1 ?

Como critérios de inclusão considerou-se elegíveis os estudos randomizados e controlados, publicados no período de janeiro de 2013 a março de 2017; em língua inglesa e em revistas com revisão por pares, com fator de impacto e disponibilizados na íntegra através do portal Coordenação de Aperfeiçoamento de Pessoal de Nível Superior (CAPES nas bases de dados Scopus (Base de dados de referências e citações), Web of Science (Science Citation Indexes), PubMed (National Library of Medicine) e MEDLINE (Sistema Online de Busca e Análise de Literatura Médica).

Foram incluídos todos os estudos que avaliaram o seguimento do controle glicêmico em portadores de diabetes mellitus tipo 2 e IMC abaixo de $35 \mathrm{~kg} / \mathrm{m}^{2}$ após a realização de cirurgia bariátrica. Artigos que aparecerem em mais de um banco de dados serão incluídos apenas uma vez, dando prioridade à base de dados Scopus.

Os critérios de exclusão foram aqueles que abordassem o seguimento controle da diabetes em pacientes com IMC acima de $35 \mathrm{~kg} / \mathrm{m}^{2}$ após a realização de cirurgia bariátrica, artigos repetidos ou que estivesse foram do período estabelecido. A pesquisa dos estudos foi realizada com base nos seguintes descritores acessados pelo MeSH: "bariatric", "surgery", "diabetes"; além do uso das palavras-chave: "Type 2 diabetes" e "bariatric surgery"; e no cruzamento das palavras foi adotada a expressão booleana "AND" e "OR". As bases de dados acessadas para as pesquisas foram SCOPUS, Web Of Science e PubMed.

Os estudos que foram considerados relevantes para serem incluídos na revisão sistemática foram escolhidos a partir da análise da leitura dos títulos, resumos e do texto integral quando o resumo não fosse suficiente para proporcionar a utilização dos critérios de inclusão ou exclusão. Vale salientar que um só investigador extraiu os seguintes dados: nome dos autores, título do artigo e ano de publicação; desenho do estudo; população estudada e principais resultados. Foi realizada de forma ativa a busca manual (handsearching) por literatura cinzenta (relatórios e/ou documentos governamentais, dissertações e/ou teses, revisões bibliográficas, anais científicos, livros com pertinência teórica na área), além da utilização de artigos de revisão não elegíveis segundo os critérios de busca para o estudo, a fim de serem referenciados no trabalho para se realizar um embasamento teórico sobre o tema.

\section{RESULTADOS}

A Figura 1 demonstra o resumo do processo metodológico de busca de evidências para fundamentação da revisão. É possível avaliar a estratégia da busca dos artigos, identificando a quantidade de artigos encontrados, suas bases de dados, como também a quantidade de artigos escolhidos após a análise final. 
Figura 1 - Fluxograma do processo metodológico de busca de evidências.

\section{SCOPU S, Web of Science, PubMed \\ MeSh TERMS: 'bariatric', 'surgery', 'diabetes', 'type 2 diabetes', 'bariatric surgery' Texto complete em inglês 2013 - Presente Artigos originais}

\section{Encontrados:}

1.204 documentos

Repetidos: 815 documentos

For a do tema: 289 documentos

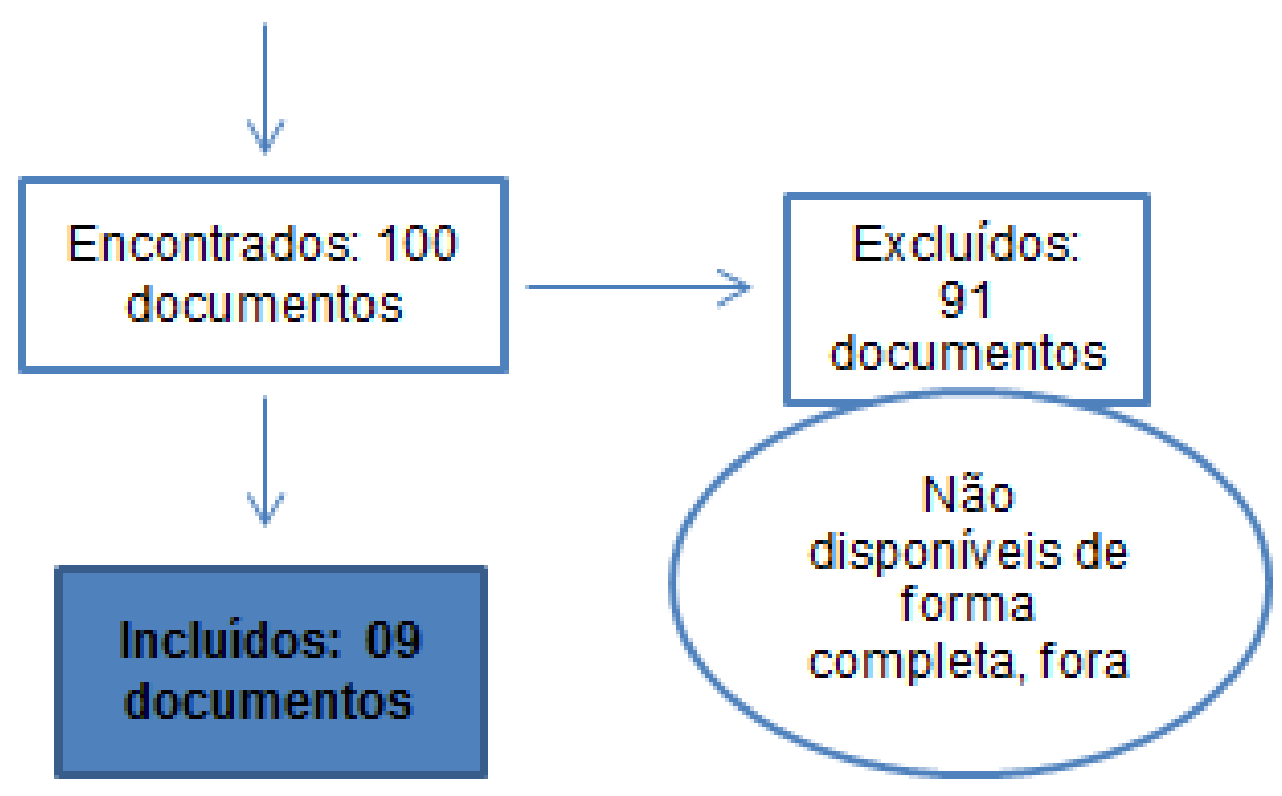

Fonte: Magalhaes FB, et al., 2019.

Identificou-se 1204 registros, dos quais 815 eram repetidos e 289 fugiam do tema, ficando 100 artigos. Na pesquisa, após a leitura dos títulos e resumos foram escolhidas 9 referências consideradas relevantes, das quais se obteve o artigo completo para leitura integral. Através da leitura detalhada dos estudos e pela confirmação de elegibilidade, os 9 artigos foram inclusos como evidências. Foi então produzida uma tabela para síntese das evidencias dos dados (Tabela 1). 
Quadro 1 - Lista de Evidencias elegíveis.

\begin{tabular}{|c|c|c|c|c|}
\hline Autor & Título & Ano & Revista & Síntese das evidências \\
\hline DIXON JB, et al & $\begin{array}{c}\text { Bypass gástrico no diabetes tipo } 2 \text { com IMC } \\
<30: \text { peso e perda de peso têm uma grande } \\
\text { influência nos resultados }\end{array}$ & 2013 & $\begin{array}{l}\text { Diabetic } \\
\text { Medicine }\end{array}$ & $\begin{array}{l}\text { Estudo prospectivo e longitudinal que avalia } 103 \text { pacientes na Coreia e em } \\
\text { Taiwan, com DM2 e IMC }<30 \mathrm{Kg} / \mathrm{m}^{2} \text {. Estes pacientes tiveram resposta } \\
\text { glicêmica melhor quanto maior o IMC basal e a remissão da doença foi } \\
\text { atribuída a perda de peso pós-cirúrgica. }\end{array}$ \\
\hline $\begin{array}{l}\text { LAKDAWALA } \\
\text { MSM, et al }\end{array}$ & $\begin{array}{l}\text { O desvio gástrico em Y de Roux permanece } \\
\text { o teste do tempo: resultados de } 5 \text { anos em } \\
\text { baixo índice de massa corporal ( } 30-35 \mathrm{~kg} / \\
\mathrm{m} \text { 2) Pacientes indianos com diabetes } \\
\text { mellitus tipo } 2\end{array}$ & 2013 & $\begin{array}{l}\text { Surgery for } \\
\text { Obesity } \\
\text { and } \\
\text { Related } \\
\text { Diseases }\end{array}$ & $\begin{array}{l}\text { Este estudo avalia os resultados em longo termo em pacientes diabéticos } \\
\text { submetidos à cirurgia de Y de Roux com IMC entre } 30 \text { e } 25 \mathrm{Kg} / \mathrm{m}^{2} \text { na Índia. } \\
\text { Afirma que o procedimento é eficaz e custo-efetivo para o tratamento de DM2 } \\
\text { não controlada. Seus preditores de sucesso foram: DM2 de início precoce, } \\
\text { maior perda de peso e maiores índices de Peptídeo-C. Associa, ainda, que } \\
\text { as melhoras após a cirurgia em hiperglicemia, hipertensão e dislipidemia } \\
\text { ajudaram a controlar a ocorrência de complicações micro e macrovasculares, } \\
\text { e diminuíram a morbidade e mortalidade da DM2. }\end{array}$ \\
\hline BOZA C, et al. & $\begin{array}{l}\text { Cirurgia Metabólica: Bypass Gástrico em Y } \\
\text { de Roux e Variáveis Associadas à } \\
\text { Remissão de Diabetes em Pacientes com } \\
\qquad \text { IMC }<35\end{array}$ & 2014 & $\begin{array}{l}\text { Obesity } \\
\text { Surgery }\end{array}$ & $\begin{array}{l}\text { Este estudo avalia os efeitos da cirurgia em Y de Roux em } 100 \text { pacientes } \\
\text { diabéticos com IMC }<35 \mathrm{Kg} / \mathrm{m}^{2} \text {, dos anos } 2002 \text { a } 2010 \text { em Santiago, Chile. } \\
\text { Confirma que o Bypass Gástrico em Y de Roux por laparoscopia é confiável } \\
\text { e efetivo em pacientes com DM2 e IMC abaixo de } 35 \mathrm{Kg} / \mathrm{m}^{2} \text { em um follow-up } \\
\text { de } 3 \text { anos. Seus melhores resultados foram vistos em pacientes não insulino- } \\
\text { dependentes. }\end{array}$ \\
\hline $\begin{array}{l}\text { SCOPINARO N, } \\
\text { et al }\end{array}$ & $\begin{array}{l}\text { Efeitos do bypass gástrico na diabetes tipo } \\
2 \text { em pacientes com IMC } 30 \text { a } 35\end{array}$ & 2014 & $\begin{array}{l}\text { Obesity } \\
\text { Surgery }\end{array}$ & $\begin{array}{l}\text { Investiga os efeitos do Bypass Gástrico em } 20 \text { pacientes diabéticos, com pelo } \\
\text { menos } 3 \text { anos de doença, IMC entre } 30 \text { e } 35 \mathrm{Kg} / \mathrm{m}^{2} \text { e } \mathrm{HbA} 1 \mathrm{c} \geq 7,5 \% \text {. Obteve } \\
\text { resultados menos estimulantes que outros estudos reportados, } \\
\text { aparentemente por ter diferentes critérios de seleçãa. Não corrobora ou } \\
\text { sustenta que efeitos independentes da perda de peso em pacientes } \\
\text { submetidos à cirurgia de Y de Roux melhorem a função das células beta em } \\
\text { pacientes com DM2 e IMC entre } 30-35 \mathrm{Kg} / \mathrm{m}^{2} \text {. }\end{array}$ \\
\hline $\begin{array}{l}\text { WENTWORTH } \\
\text { JM }\end{array}$ & $\begin{array}{l}\text { Cuidados multidisciplinares com diabetes } \\
\text { com e sem cirurgia bariátrica em pessoas } \\
\text { com sobrepeso: um estudo controlado } \\
\text { randomizado }\end{array}$ & 2014 & $\begin{array}{l}\text { Lancet } \\
\text { Diabetes } \\
\text { Endocrinol }\end{array}$ & $\begin{array}{l}\text { Estudo conduzido em Melbourne, Australia, aberto, controlado e } \\
\text { randomizado entre } 2009 \text { e } 2013 \text {, compara pacientes com DM2 obesos (IMC } \\
\text { entre 25-30) em tratamento multidisciplinar sozinho ou com cirurgia de banda } \\
\text { gástrica. Seu resultado mostra remissão de diabetes após } 2 \text { anos nos } \\
\text { pacientes submetidos à cirurgia com efeitos adversos aceitáveis, sendo uma } \\
\text { opção de tratamento para diabetes nesta população. }\end{array}$ \\
\hline
\end{tabular}




\begin{tabular}{|c|c|c|c|c|}
\hline HSU CC & $\begin{array}{l}\text { Efeito da cirurgia bariátrica versus } \\
\text { tratamento médico no diabetes tipo } 2 \text { em } \\
\text { pacientes com índice de massa corporal } \\
\text { inferior a 35: resultados em cinco anos. } \\
\text { Outcomes }\end{array}$ & 2015 & $\begin{array}{l}\text { Journal of } \\
\text { the } \\
\text { American } \\
\text { Medical } \\
\text { Association }\end{array}$ & $\begin{array}{l}\text { Compara a eficácia em } 5 \text { anos entre a cirurgia gástrica metabólica e o } \\
\text { tratamento medicamentoso para controle glicêmico em pacientes com DM2 e } \\
\text { IMC }<35 \mathrm{Kg} / \mathrm{m}^{2} \text {. Corrobora com achados que a melhora do controle glicêmico } \\
\text { permanece até os cinco anos, ao menos, mas não estabelece os benefícios } \\
\text { em mortalidade e morbidade, pois necessita de acompanhamento a longo } \\
\text { termo maior. }\end{array}$ \\
\hline KULAR KS & $\begin{array}{l}\text { Sete anos de desvio mini-gástrico em } \\
\text { pacientes com diabetes tipo II com índice } \\
\text { de massa corporal < }<5 \mathrm{~kg} / \mathrm{m} 2\end{array}$ & 2015 & $\begin{array}{l}\text { Obesity } \\
\text { Surgery }\end{array}$ & $\begin{array}{l}\text { Pesquisa que avaliou os resultados após } 7 \text { anos do Bypass Gástrico em } 128 \\
\text { pacientes diabéticos com IMC entre } 30 \text { e } 35 \mathrm{Kg} / \mathrm{m}^{2} \text {, demonstrando um bom } \\
\text { controle a longo prazo do DM2 em pacientes com obesidade grau I. Avaliou } \\
\text { ainda que a intervenção precoce está associada com melhores níveis de } \\
\text { remissão. }\end{array}$ \\
\hline PETRY TZ & $\begin{array}{l}\text { Efeito da cirurgia de bypass duodenal- } \\
\text { jejunal no controle glicêmico no diabetes } \\
\text { tipo 2: um estudo controlado randomizado. }\end{array}$ & 2015 & OBESITY & $\begin{array}{l}\text { Estudo conduzido em } 17 \text { pacientes com sobrepeso e obesidade e diabetes } \\
\text { tipo 2, randomizados para o tratamento medicamentoso ou Bypass Duodeno- } \\
\text { Jejunal. Conclui que a cirurgia promove perda de peso moderada e melhora } \\
\text { a função metabólica em pacientes com DM2, no entanto o estudo não pode } \\
\text { separar os benefícios da perda de peso com o potencial efeito terapêutico do } \\
\text { próprio bypass no trato gastrointestinal nas melhorias metabólicas } \\
\text { observadas. }\end{array}$ \\
\hline $\begin{array}{c}\text { WENTWORTH } \\
\text { JM }\end{array}$ & $\begin{array}{l}\text { Cirurgia de banda gástrica leva a melhora } \\
\text { da secreção de insulina em pessoas com } \\
\text { sobrepeso com diabetes tipo } 2\end{array}$ & 2015 & $\begin{array}{l}\text { Obesity } \\
\text { Surgery }\end{array}$ & $\begin{array}{l}\text { Ao estudar } 44 \text { pacientes, com IMC entre } 25 \text { e } 30 \mathrm{Kg} / \mathrm{m}^{2} \text { conclui que em } \\
\text { pacientes com diabetes a cirurgia laparoscópica de banda gástrica melhora a } \\
\text { função das células beta pancreáticas após } 02 \text { anos. }\end{array}$ \\
\hline
\end{tabular}




\section{DISCUSSÃO}

Os atuais estudos sobre tratamento cirúrgico em diabéticos tipo 2 emergem com a idealização da possibilidade de uma cirurgia metabólica para todos os pacientes com descontrole glicêmico apesar da otimização de drogas antidiabéticas e mudanças no estilo de vida. Hoje, a permissão do uso da bariátrica apenas é estabelecido para pacientes com IMC $\geq 35 \mathrm{~kg} / \mathrm{m}^{2}$ que possuem DM2 como comorbidade de acordo com a American Diabetes Association (ADA) e Federação Internacional de Diabetes (IDF). Faz-se então necessário estudos com amostragem diversificada de pacientes com sobrepeso e obesidade, variando-se gênero, idade, tempo e controle glicêmico para obter resposta fidedigna sobre referendar a cirurgia bariátrica como opção viável e plausível para controle e até mesmo remissão da diabetes (RUBINO F e AMIEL SA, 2014).

Para confirmar a remissão da diabetes, de acordo com a Federação Internacional de Diabetes, a Hemoglobina A1c deve ser menor que $42 \mathrm{mmol} / \mathrm{mol}$ (milimol é a milésima parte de um mol) (6\%) (IDF TASKFORCE ON EPIDEMIOLOGY AND PREVENTION), segundo Rubino F e Amiel SA (2014) deve-se escolher pacientes com início recente de DM2 e boa função residual de células beta para se obter uma maior e sustentada taxa de remissão de DM2.

É possível observar que tratamentos cirúrgicos que fazem by-pass no trato gastrointestinal superior são efetivos em alcançar remissão parcial ou completa do DM2, sugerindo que há efeitos de controle glicêmicos independentes do emagrecimento. Os eventos relatados em associação com a cirurgia bariátrica para remissão do DM2 são: sensibilidade multiorgânica à insulina, função das células beta, resposta da incretina, microbioma intestinal, metabolismo da bile ácida, metabolismo intestinal da glicose e 'browning' de tecido adiposo (CHONDRONIKOLA M, et al., 2016).

Mecanismos estes que necessitam de mais estudos para determinar sua importância em pacientes com DM2, apesar da evidência que a cirurgia induz efeitos antidiabéticos rápidos, independente da perda de peso (RUBINO F e AMIEL SA, 2014).

As cirurgias gástricas apontadas como possibilidade para o tratamento são: Bypass gástrico em $Y$ de Roux, Gastrectomia vertical com interposição ileal laparoscópica, Banda gástrica ajustável laparoscópica e a Derivação bilio-pancreática (CHONDRONIKOLA M, et al., 2016).

De acordo com o levantamento de dados (Tabela 1) a cirurgia mista, com componentes restritivos e absortivos, o chamado by-pass, é a realizada mais comumente em pacientes obesos. Tem maior sucesso na perda de peso quando comparada a técnica de banda gástrica e menores riscos, como a necessidade de réoperação.

O aumento da massa das células beta também foi uma hipótese levantada após descrições controversas de nesidioblastose como complicação do $Y$ de Roux, ou das observações de aumento nos níveis de fator promotor da Insulina (IPF1) e prevenção de perdas de células beta após experimento em roedores com bypass duodeno-jejunal, como também relato de casos de massa pancreática heterotópica após $Y$ de Roux em humanos. Tanto em roedores quanto humanos, $Y$ de Roux aumenta os níveis pós-prandiais de peptídeo semelhante a glucagon 1 (GLP-1), uma incretina que estimula a liberação de insulina pelo pâncreas e exerce efeitos antiapoptóticos nas células beta (RUBINO F e AMIEL SA, 2014).

As incretinas são hormônios gastrointestinais liberados em resposta à alimentação, apresentam papel central na regulação pós-prandial de insulina, parecem fornecer a base da compreensão da alta perda de peso da cirurgia bariátrica. GLP-1 regula o peso corporal por inibir o apetite e atrasar ações gástricas e esvaziamento que dependem de ativação no sistema nervoso central por receptores de GLP-1 (JOÃO AL, et al., 2016).

A teoria anti-incretina relata que além dos efeitos da GLP-1, a passagem de nutrientes no trato gastrointestinal pode também causar ativação de mecanismo de feedback negativo (anti-incretina) para prevenir hipoglicemia hiperinsulínica pós-prandial, necessária também para prevenir proliferação descontrolada de células beta (RUBINO F e AMIEL SA, 2014). 
Após inúmeros estudos e trials randomizados comparando o manejo medicamentoso de DM2 com o cirúrgico, confirmou-se que cirurgia bariátrica é mais efetiva no tratamento de DM2 (GANGULY S, et al., 2015).

No estudo realizado por Wentworth JM et al. (2014) em Melbourne na Austrália, os pacientes submetidos à tratamento multidisciplinar associado à banda gástrica por diabetes e sobrepeso (IMC entre 25 e $30 \mathrm{Kg} / \mathrm{m}^{2}$ ) apresentaram melhora no controle glicêmico tendo $91 \%$ da amostragem uma $\mathrm{HbA} 1 \mathrm{c}<7 \%$ ao fim de dois anos de seguimento, como também seus efeitos adversos foram aceitáveis: 17\% apresentaram intolerância alimentar com necessidade de redução do volume da banda, $17 \%$ necessitaram de cirurgias não planejadas durante o tratamento, e um paciente num total de 51 necessitou de revisão cirúrgica; Corrobora então para a eficácia da cirurgia laparoscópica de banda gástrica ajustável para o tratamento de diabetes em pacientes com sobrepeso.

O mesmo grupo de Wentworth JM et al., (2015) conduziu uma nova pesquisa em 44 pacientes diabéticos com sobrepeso, IMC entre 25 e $30 \mathrm{Kg} / \mathrm{m}^{2}$, submetidos à Banda Gástrica para correlacionar a atividade basal de sua função beta-pancreática e os resultados glicêmicos após 02 anos de acompanhamento, concluiu que a resposta insulínica não se modificou, no entanto a relação Peptídio-C/insulina em jejum aumentou, portanto, a função basal das células beta se correlacionou com a remissão da diabetes mas não com a redução da HbA1c após a cirurgia. Sugere, ainda, que novos estudos se fazem necessários para determinar a duração desta recuperação beta-pancreática e melhora glicêmica após a cirurgia de Banda Gástrica.

Segundo Petry TZ et al., (2015) ao realizarem um estudo controlado e randomizado numa amostragem de 17 pacientes diabéticos com sobrepeso e obesidade com IMC $\leq 35 \mathrm{Kg} / \mathrm{m}^{2}$. O grupo controle foi submetido ao tratamento padrão para controle glicêmico e o outro grupo foi submetido ao bypass duodenojejunal com mínima ressecção gástrica. Em comparação ao grupo controle a cirurgia ofereceu perda de peso progressiva, melhora no teste oral de tolerância à glicose, sensibilidade insulínica e controle glicêmico geral e com uso de menos drogas hipoglicemiantes. No entanto, não foi possível separar os benefícios da moderada perda de peso do potencial terapêutico do próprio by-pass no trato gastrointestinal nas melhorias metabólicas observadas.

Kular KS et al.,(2016) descreveram os resultados em 128 portadores de diabetes com IMC entre 30 e $35 \mathrm{Kg} / \mathrm{m}^{2}$, após sete anos de experiência, com o mini bypass gástrico e evidenciaram que após seis meses do procedimento $95 \%$ dos pacientes já apresentavam uma $\mathrm{HbA} 1 \mathrm{c}<7 \%$, ao findar de sete anos a taxa de remissão completa da doença foi de $53 \%$ e apenas 2 pacientes (1,6\%) apresentaram complicações (sangramento em grampeamento cirúrgico e cetoacidose diabética). Concluíram ainda que o mini by-pass se relaciona a um bom controle em longo prazo do diabetes tipo 2 em pacientes com obesidade grau I, e quanto mais precoce a intervenção maiores serão as taxas de remissão.

Dixon JB et al., (2012) avaliaram 103 pacientes diabéticos com $\mathrm{IMC}<30 \mathrm{Kg} / \mathrm{m}^{2}$, na Coreia e em Taiwan, submetidos ao by-pass em $Y$ de Roux e mostraram que 30\% obtiveram controle glicêmico com $\mathrm{HbA} 1 \mathrm{c} \leq 6 \%$ em um ano de seguimento e pacientes com diabetes a menos de sete anos e IMC $>27 \mathrm{Kg} / \mathrm{m}^{2}$ seriam os melhores respondedores à tecnica. Neste caso um $\mathrm{IMC}<27 \mathrm{~kg} / \mathrm{m}^{2}$ e níveis de Peptídeo-C $<2,0 \mathrm{ng} / \mathrm{mL}$ foram considerados preditores de uma resposta glicêmica pobre. Os pacientes que apresentaram maiores perdas ponderais e os que possuíam Peptídio-C $>2,4 \mathrm{ng} / \mathrm{mL}$ foram os que atingiram o melhor controle glicêmico.

De acordo com Lakdawala MSM et al., (2013) eles publicaram um trabalho envolvendo 52 pacientes indianos com IMC entre $30-35 \mathrm{Kg} / \mathrm{m}^{2}$ e diabetes não controlado submetidos à cirurgia em $\mathrm{Y}$ de Roux. Após cinco anos de seguimento a porcentagem de perda de peso foi de $67,8 \%, 84,6 \%$ dos pacientes atingiram a normoglicemia e $73,1 \%$ alcançaram a remissão completa em um ano. Novo ganho de peso ocorreu em 8 pacientes. Após cinco anos a taxa de remissão completa caiu para $57,7 \%$, no entanto $96,2 \%$ melhoraram seus status metabólicos (hipertensão e dislipidemia) confirmando que a cirurgia laparoscópica em Y-de-Roux é segura, eficaz e custo-efetiva para pacientes diabéticos com obesidade grau I. Os preditores de sucesso foram: início precoce da DM2, maior perda de peso e maiores níveis de Peptídio-C basal.

Através de estudo retrospectivo conduzido na Universidade Católica do Chile, Boza C et al. (2014) avaliaram 100 pacientes diabéticos com $\mathrm{IMC}<35 \mathrm{Kg} / \mathrm{m}^{2}$ submetidos ao Bypass gástrico em $\mathrm{Y}$-de-Roux e 
demonstraram completa remissão do diabetes em $53,2 \%$ da amostragem e nenhuma melhora em $11,7 \%$, com um odds ratio $15,1(1,8-81,1)$ e $p=0,002$, sendo seus melhores resultados em pacientes que não faziam uso de insulinoterapia.

Já na Universidade de Ganoa, na Itália, um estudo com pacientes diabéticos com IMC entre 30 e $35 \mathrm{Kg} / \mathrm{m}^{2}$ (SCOPINARO $\mathrm{N}$ et al., 2014) investigou os benefícios do controle glicêmico após a cirurgia de Y-de-Roux monitorados por 3 anos e observaram uma remissão de diabetes em $25 \%$ dos casos, controle em $45 \%$ e melhora em todos os demais. A remissão se correlacionou negativamente com o tempo de diabetes e positivamente com o Peptídio-C. Apesar de seus resultados positivos, para Scopinaro N et al., (2014) estes não foram tão bons quanto os já descritos em literatura, não corroborando com a ideia de efeitos benéficos na função beta-pancreática que sejam independentes da perda de peso pós-cirúrgica em pacientes com IMC de 30 a $35 \mathrm{Kg} / \mathrm{m}^{2}$.

Hsu CC et al., (2015) conduziram um estudo de coorte prospectivo de cinco anos com avaliação de pacientes diabéticos com $\mathrm{IMC}$ até $35 \mathrm{Kg} / \mathrm{m}^{2}$ submetidos à cirurgia metabólica (by-pass gástrico ou gastrectomia vertical), comparando-os à uma população apenas com tratamento medicamentoso que se equivalem em idade, IMC e duração da diabetes. Em seus resultados o grupo cirúrgico apresentou uma média de $21 \%$ de perda de peso, HbA1c médio caiu de $9,1 \%$ para $6,3 \%$, sendo $36 \%$ com remissão completa da doença $(\mathrm{HbA} 1 \mathrm{c}<6 \%)$ e $14 \%$ remissão parcial (HbA1c entre 6 e 6,5\%), sendo estas reduções significantemente maiores quando comparado ao grupo apenas medicado (tudo $\mathrm{P}<.001$ ). Comprova, portanto, que os efeitos benéficos da cirurgia nesta população dura ao menos 5 anos, mas os benefícios medidos em mortalidade e morbidade necessitariam de maior tempo de estudo.

Por fim Cummings $D$ et al., (2016) iniciaram um estudo multicêntrico e controlado com a proposta de comparar, em um período de 8 anos, pacientes insulino-dependentes submetidos à cirurgia de $\mathrm{Y}$-de-Roux ou ao tratamento medicamentoso padrão para DM2 e definir seus índices de morbidade e mortalidade associados ao diabetes.

\section{CONSIDERAÇÕES FINAIS}

Após levantamento de dados e apoiando-se apenas nos estudos originais que abordam o tratamento cirúrgico do diabetes tipo 2 em pacientes com $I M C<35 \mathrm{Kg} / \mathrm{m}^{2}$, um total de nove artigos finalizados, concluímos que apesar dos indícios de bons resultados, com remissão de níveis glicêmicos, controle de morbidade e mortalidade devido às complicações da DM2, é um tema que necessita de maiores pesquisas, com amostragem multicêntrica e comparável aos estudos que apoiam o uso da cirurgia para tratamento de pacientes com $I M C>35 \mathrm{Kg} / \mathrm{m}^{2}$. As pesquisas sobre o tema são recentes, sendo a mais longa efetuada num período de sete anos, seus resultados no geral mostram excelentes taxas de controle no primeiro ano de seguimento, porém conforme o passar do tempo os seus resultados decaem, não sendo possível efetivamente corroborar com seus achados iniciais.

\section{REFERÊNCIAS}

1. BOZA C, et al. Metabolic surgery: roux-en-Y gastric bypass and variables associated with diabetes remission in patients with $\mathrm{BMI}<35$. Obesity surgery, 2014; 24(8):1391-1397.

2. BRASIL. MINISTÉRIO DA SAÚDE. (Org.). Vigilância de Fatores de Risco e Proteção para Doenças Crônicas por $\begin{array}{lllll}\text { Inquérito } & \text { Telefônico } & \text { VIGITEL. } & 2016 . & \text { Disponível }\end{array}$ <https://www.endocrino.org.br/media/uploads/PDFs/vigitel.pdf>. Acesso em: 08 maio 2017.

3. CATOI AF, et al. Metabolic Mechanism in obesity and type 2 diabetes: Insights from Bariatric/Metabolic Surgery. Obesity Facts, 2015; 8:350-363.

4. CHONDRONIKOLA M, et al. Bariatric surgery and type 2 diabetes: are there weight loss- independent therapeutic effects of upper gastrointestinal bypass? Journal of Internal Medicine, 2016; 280: 476-486.

5. COELHO D, et al. Diabetes remission rate in different BMI grades following Roux-en-Y gastric bypass. ABCD. Arquivos Brasileiros de Cirurgia Digestiva (São Paulo), 2018; 31(1). 
6. CUMMINGS D, et al. Gastric bypass surgery vs intensive lifestyle and medical intervention for type 2 diabetes: the CROSSROADS randomised controlled trial. Diabetologia, 2016; 59(5): 945-953.

7. $\mathrm{DIXON} J \mathrm{~B}$, et al. Gastric bypass in type 2 diabetes with $\mathrm{BMl}<30$ : weight and weight loss have a major influence on outcomes. Diabetic Medicine, 2013; 30(4):127-134.

8. ELIGAR VS, NARAYANASWAMY AKP. Bariatric surgery and remission of type 2 diabetes mellitus. Current Opinion Endocrinology Diabetes Obesity,2016; 27:97-99.

9. GANGULY S, et al. Metabolic bariatric surgery and type 2 diabetes mellitus: an endocrinologist's perspective. The Journal of Biomedical Research, 2015; 29(2) 105-111.

10. HSU CC, et al. Effect of bariatric surgery vs medical treatment on type 2 diabetes in patients with body mass index lower tha 35: five year outcomes. JAMA Surgery, 2015; 150(12):1117-1124.

11. JOÃO AL, et al. The incretin system ABCs in obesity and diabetes - novel therapeutic strategies for weight loss and beyond. Obesity Reviews, 2016; 17:553-572.

12. KULAR KS, et al. Seven years of mini-gastric by-pass in type II diabetes patients with body mass index $<35 \mathrm{~kg} / \mathrm{m}^{2}$. Obesity surgery, 2016; 26(7): 1457-1462.

13. LAKDAWALA MSM, et al. Roux-en-Y gastric bypass stands the test of time: 5 -year results in low body mass index $\left(30-35 \mathrm{Kg} / \mathrm{m}^{2}\right)$ Indian patients with type 2 diabetes mellitus. Surgery for obesity and related diseases, 2013; 9: 370378.

14. MALKANI S. An update on the role of bariatric surgery in diabetes management. Current Opinion Endocrinology Diabetes Obesity, 2015; 22: 98-105.

15. PETRY TZ, et al. Effect of Duodenal-Jejunal Bypass Surgery on Glycemic control in type 2 diabetes: A randomized Controlled Trial. Clinical Trials and Investigations, 2015; (23)1973-1979.

16. RUBINO FE, AMIEL SA. Is the gut the "Sweet Spot" for the Treatment of Diabetes. Diabetes, 2014; 63: 2225-2228.

17. SCOPINARO N, et al. Effects of Gastric Bypass on type 2 diabetes in patients with BMI 30 to 35 . Obesity surgery, $2014 ; 24(7): 1036-1043$.

18. WENTWORTH JM, et al. Multidisciplinary diabetes care with and without bariatric sugery in overweight people: a randomized controlled trial. The lancet Diabetes \& endocrinology, 2014; 2(7): 545-552.

19. WENTWORTH JM, et al. Gastric Band Surgery Leads to Improved Insulin Secretion n Overweight People With Type 2 Diabetes. Obesity Surgery, 2015; 25: 2400-2407. 